\title{
Multiresponse Optimization for a Novel Compliant Z-Stage by a Hybridization of Response Surface Method and Whale Optimization Algorithm
}

\author{
Minh Phung Dang, ${ }^{1}$ Hieu Giang Le, ${ }^{1}$ Ngoc N. Trung Le, ${ }^{1}$ Ngoc Le Chau, ${ }^{2}$ \\ and Thanh-Phong Dao $\mathbb{B D}^{3,4}$ \\ ${ }^{1}$ Faculty of Mechanical Engineering, Ho Chi Minh City University of Technology and Education, Ho Chi Minh City, Vietnam \\ ${ }^{2}$ Faculty of Mechanical Engineering, Industrial University of Ho Chi Minh City, Ho Chi Minh City, Vietnam \\ ${ }^{3}$ Division of Computational Mechatronics, Institute for Computational Science, Ton Duc Thang University, \\ Ho Chi Minh City, Vietnam \\ ${ }^{4}$ Faculty of Electrical \& Electronics Engineering, Ton Duc Thang University, Ho Chi Minh City, Vietnam
}

Correspondence should be addressed to Thanh-Phong Dao; daothanhphong@tdtu.edu.vn

Received 16 March 2021; Revised 5 April 2021; Accepted 11 April 2021; Published 23 April 2021

Academic Editor: Dr. Dilbag Singh

Copyright (C 2021 Minh Phung Dang et al. This is an open access article distributed under the Creative Commons Attribution License, which permits unrestricted use, distribution, and reproduction in any medium, provided the original work is properly cited.

\begin{abstract}
A novel compliant z-stage is applied for positioning and indenting a specimen in nano/microindentation testing system. For an excellent operation, the proposed z-stage can concurrently satisfy multicriteria comprising high safety factor, small parasitic motion, and large output displacement. The key aims of this article are to present a novel design of the compliant $\mathrm{z}$-stage as well as an effective integration methodology of Taguchi method, response surface method, weight factor calculation based on signal to noise, and the whale optimization algorithm to resolve a design optimal problem so as to enrich the quality performances of the proposed stage. Primarily, the z-stage is designed based on four-lever amplifier, compliant hinge shifted arrangement mechanism, zigzag-based flexure spring guiding mechanism, and symmetric six leaf hinges-based parallel guiding mechanism. Secondly, the number experiment data are achieved by the Taguchi method and finite element analysis. Subsequently, the regression functions among input variables and quality characteristics are formed by exploiting response surface method. In addition, the weight factors for every characteristic are defined. Additionally, the sensitivity analysis is accomplished for determining influences of input variables on quality responses. Ultimately, based on regression equations, the whale optimization algorithm is executed to define the optimal factors. The consequences indicated that the output deformation is about $454.55 \mu \mathrm{m}$ and the safety factor is around 2.38. Furthermore, the errors among the optimal consequences and the confirmations for the safety factor and output deformation are $7.12 \%$ and $4.25 \%$, correspondingly. By using Wilcoxon and Friedman methods, the results revealed that the proposed algorithm is better than the cuckoo search algorithm. Based on the quality convergence characteristics of hybrid approach, the proposed method is proficient for resolving complicated multiobjective optimization.
\end{abstract}

\section{Introduction}

Nano/microindentation testing technique has been utilized for supplying small displacement or force for measuring the depth in the nano/microratio quality characteristics of material specimen. This technology is utilized to monitor mechanical features of various materials $[1,2]$. However, entire positioner utilized conventional joints to connect mechanical components together. Consequently, there is a complexity for obtaining precise positioning due to the demerits of backlash, friction as well as abrasion. As a result, an accurate positioner is crucial for locating position as well as checking material specimen.

Based on the essential merits of compliant mechanism such as free wear, free backlash, light weight, small friction, high precision, low cost, and compact mechanism [3-5], it 
has been extensively employed to discover and gradually replace traditional mechanism. In addition, accurate positioning platforms are also inherited from quality characteristic benefits of the compliant mechanism, and they are widely utilized for potential applications such as constantforce precision positioning [6], ultra-violet nanoimprint lithography [7], atomic force microscopy [8], and positioning system for cell micromanipulation [9]. In addition, related to positioning stage for nanoindentation tester, Huang et al. proposed a novel in situ nanoindentation instrument with the maximal output displacement of about $11.44 \mu \mathrm{m}$. The device can realize coarse modification and accuracy loading and unloading procedure automatically [10]. Moreover, the minimal indentation instrument with the operating stroke of $40 \mu \mathrm{m}$ for indenter was developed by Huang et al. [11].

Meanwhile, the working travel of piezoelectric (PZT) actuator is limited, so, in order to obtain enough significant output deformation and high precision for $z$-axis-indenter, the compliant positioning stages regularly combine various displacement amplifications [12]. For specifics, Chang et al. offered a Scott-Russell mechanism for expanding the stroke of PZT for gaining high deformation [13] and Wang et al. developed the displacement amplification mechanism of lever mechanism and bridge mechanism combined into compliant gripper [14]. Tang et al. proposed a new hydraulic deformation magnification structure for PZT actuator for achieving large stroke [15]. Xu proposed a novel flexure gripper according to the Scott-Russell structure to magnify output displacement [16], and Kim et al. exploited a double amplifier comprising two L-shape levers in a compliant 3DOF stage [17]. Although there have been a lot of compliant mechanisms with one degree of freedom, there has been a little attention in developing a compliant $\mathrm{z}$-stage for use in nanoindentation devices. Furthermore, there is a need to develop a new z-stage with full consideration of multiple functions, simultaneously. In order to fill in the gaps in the literatures, the present paper is aimed to design a novel compliant $\mathrm{z}$-stage based on the four-lever amplification mechanism, compliant hinge shifted arrangement mechanism, zigzag-based flexure spring guiding mechanism, and symmetric six leaf hinges-based parallel guiding mechanism.

In order to reach multiple good functions for the $z$-stage, an integrated method of the Taguchi method (TM), response surface method (RSM), and whale optimization algorithm [18] is developed to optimize main geometric parameters of the proposed z-stage. Commonly, the output deformation and the safety factor are the greatest essential features. However, two characteristics are struggled together. As a result, in order to balance and achieve the desires of a large output deformation as well as a high safety factor, simultaneously, the hybrid method is proposed for conducting the optimal problem. Additionally, the key geometric factors of $z$-stage were optimized for achieving aforementioned requests.

Generally, the TM is widespread exploited in optimal analysis engineering for generating initial numerical data [19]. By contrast, in order to perform multiresponse optimization, the TM should integrate with various algorithms.
In addition, in previous studies, Canan Kandilli et al. employed Ansys workbench to optimize the design and operation factors of a PVT system integrated with natural zeolite [20]. Maryam Safa et al. used the adaptive neurofuzzy inference system (ANFIS) approach to predict the highest significant factors on vectorial crystal growth of extremely oriented vertically aligned carbon nanotubes [21]. Chunbiao Zhu et al. utilized a saliency algorithm to create a significance map for guiding the style transmitting procedure. Zeeshan Ali et al. utilized complex neutrosophic set (CNS) to instigate the novel dice similarity measures and generalized dice similarity [22]. Furthermore, several population-based algorithms [23-25] were developed. For example, Golpîra et al. applied the genetic algorithm (GA) for optimization key factors of the power system [26], Basu et al. utilized the cuckoo search algorithm for solving economic dispatch [27], and Chander et al. proposed particle swarm optimization for optimizing multilevel thresholding [28]. Prakash et al. utilized the multiobjective particle swarm optimization (MO-PSO) method to define the electrical discharge machining (EDM) factors [29]. In contrast, these algorithms demand primary control factors. Other approaches to enhance convergence velocity with less primary factors are, for instance, teaching learning-based optimization (TLBO) [30-33] and Rao's algorithms [34].

In order to conduct multicriteria, hybrid approaches such as the TM-RSM and GA [35], the TM-fuzzy based on moth-flame optimization [36], PSO-GA [37, 38], RSM-PSO [39], gravitational search algorithm (GSA)-GA [40, 41], grey-TM, RSM, and entropy measurement [42], PSO-neural network (NN) [43], TM-RSM and NSGA-II [44], and fuzzy logic-ANFIS and lightning attachment procedure optimization (LAPO) [45] have been implemented by many researchers. For more specifics, Dao proposed hybrid approach of the grey-TM according to fuzzy logic for optimizing the main parameters of a compliant stage [46]. Dang et al. developed a combined method of the TM, RSM, enhanced ANFIS, and TLBO for a positioner [47].

Meanwhile, an integration method of TM, RSM [48], and whale optimization algorithm (WOA) integrated the weight factor calculation method based on the signal to noise for optimizing a compliant $\mathrm{z}$-stage applied for the indenter in nanoindentation tester which has not been studied yet. As a result, based on the global convergence quality characteristics of the WOA, a proficient hybrid approach of TM, RSM, and WOA [18, 49-52] is developed to resolve multicriteria optimization trouble of the z-stage in this article. Additionally, the weight factors based on signal to noise [53] for each response are calculated and assigned for the total weighted objective function in order to enhance the optimal precision results.

This article proposes a new optimization design for the compliant z-stage. In order to solve the optimization, a hybridization of the TM, RSM, weight factor calculation, and whale optimization algorithm is developed. The TM-RMS is integrated with the finite element method to retrieve datasets and establish the regression equations. Based on the regression models, the whale optimization is extended to optimize the geometry factors of the z-stage. Then, the 
numerical validations are performed for confirming the optimal consequences. Furthermore, the sensitivity analysis and analysis of variance are studied for all parameters. Finally, a few statistical analysis [54] is executed for evaluating the manners of the proposed algorithm with another approach.

\section{Conceptual Design of Compliant Z-Stage}

In this study, an intended prospective application of the compliant z-stage is suggested, as illustrated in Figure 1. This system comprises a coarse $z$-stage, a coarse xy-stage, a fine $x y$-stage, and a fine $z$-stage. For specifics, the coarse xy-stage is applied for the initial coarse positioning and a fine xy-stage is used for the fine positioning the material specimen as well as the coarse $\mathrm{z}$-stage is employed for moving the sample to near the indenter. Finally, the fine $\mathrm{z}$-stage is named as the compliant $\mathrm{z}$-stage which is utilized for the fine positioning the indenter to reach to the location of material sample.

2.1. Integrated Amplification Mechanism of Displacement. A lever structure is beneficial structure for amplifying the values of force and displacement. First and foremost, Figure 2 depicts an operating principle of one lever mechanism for magnifying the displacement along the $z$ axis. For specifics, point $\mathrm{O}$ represents a fixed joint that is the rotary position of the lever. In addition, $M$ and $N$ are the input and output points, respectively. The working principle of lever mechanism is demonstrated as follows: when a vertical displacement $\Delta d_{1}$ impacts into the input point $M$, the lever will rotate a relative angle $\gamma$ around the $z$-axis. Consequently, the point $N$ moves to $N^{\prime}$ and the output displacement $\Delta d_{2}$ will obtain in vertical $y$-axis direction. In contrast, this mechanism generates easily high decoupling error. Therefore, displacement amplification mechanism with symmetric multilever is integrated to amplify the output displacement as well as reduce the parasitic motion. Later on, in order to decline more the decoupling error, a guiding mechanism with zigzag-based flexure spring and parallel guiding mechanism are integrated into the magnification mechanism, as exhibited in Figure 3. In order to decline more the error of decoupling error, parallel guiding mechanism-based symmetric six leaf hinges was integrated into a hybrid amplification mechanism. Especially, this mechanism affects significantly in reducing parasitic motion for precise z-stage. According to the lever mechanism physical features, the magnification proportion can be around gained:

$$
r_{\text {lever }}=\frac{\Delta d_{2}}{\Delta d_{1}}=\frac{d_{2}}{d_{1}} .
$$

The function of multiple levers magnifier is supposed in form as follows:

$$
r_{\text {lever }}=k \times \frac{d_{2}}{d_{1}} .
$$

For a four-lever magnification, $k$ is supposed for selecting 4 . Due to the influences of flexure hinges such as thin thickness, the rotation characteristics of different hinges, the travel loss as well as distance among continued levers, so, equation (2) is not precise for amplification ratio calculation. It should be checked by finite element analysis (FEA) to obtain the accurate consequences. Likewise, so as to increase high stiffness as well as decline decoupling error, the zigzag-based flexure springs and parallel guiding mechanism were integrated into the magnification mechanism of the z-stage.

2.2. Compliant Z-Stage. In this research, based on the great characteristics of the material Al 7075 (good yield strength of $503 \mathrm{MPa}$, Young's modulus of $71700 \mathrm{MPa}$, light density of $2770 \mathrm{~kg} / \mathrm{m}^{3}$, and Poisson's ratio of 0.33 ), this material was chosen for designing the proposed $\mathrm{z}$-stage. In addition, in order to achieve more than the working travel of $40 \mu \mathrm{m}$, the Physik-device PZT actuator (P-225.10) with a maximal stroke variety $[0,15] \mu \mathrm{m}$ or translational crew for creating more higher displacement will be utilized for generating input displacement and connected with proposed hybrid amplification mechanism. For specifics, Figures 4(a) and 4(b) illustrate the details and main geometric dimensions of the proposed compliant z-stage, respectively. The z-stage comprises following parts: (i) fifteen fixed holes are used for locating the z-positioner and (ii) a PZT actuator or translational screw can be utilized for generating the input displacement for the $\mathrm{z}$-stage. Additionally, force transferring guiding mechanism based on zigzag flexure springs and parallel guiding mechanism are integrated into the $\mathrm{z}$-stage for reducing decoupling error.

The sum dimension of $z$-stage is around $301 \mathrm{~mm} \times 162 \mathrm{~mm} \times 16 \mathrm{~mm}$. Especially, compared with common flexure hinges, elliptical hinge is proposed for utilizing so as to gain accurate rotary-center and high output displacement, simultaneously. Besides, in order to reduce decoupling error as well as gain mobility, parallel guiding mechanism is also used to integrate into z-stage. In addition, by checking initial quality responses, in order to avoid plastic failures as well as obtain a high safety factor, an input displacement of $65 \mu \mathrm{m}$ is proposed.

The proposed $\mathrm{z}$-stage would be advanced for creating linear deformation employed for in situ nanoindentation tester. Table 1 provides geometric dimensions of the z-stage. Thus, some key factors of the stage need to be considered in optimizing procedure in order to boost the quality features of the z-stage.

\section{Methodology}

3.1. Formulation of Optimal Problem. The compliant z-stage ought to satisfy the subsequent requisites: (1) the safety factor $\left(F_{1}\right)$ is expected to obtain high value in order to assure the strength of the z-stage and (2) high $z$-axis displacement $\left(F_{2}\right)$ is also demanded for gaining high value for expanding locating competence to position as well as indent for checking a material specimen. 


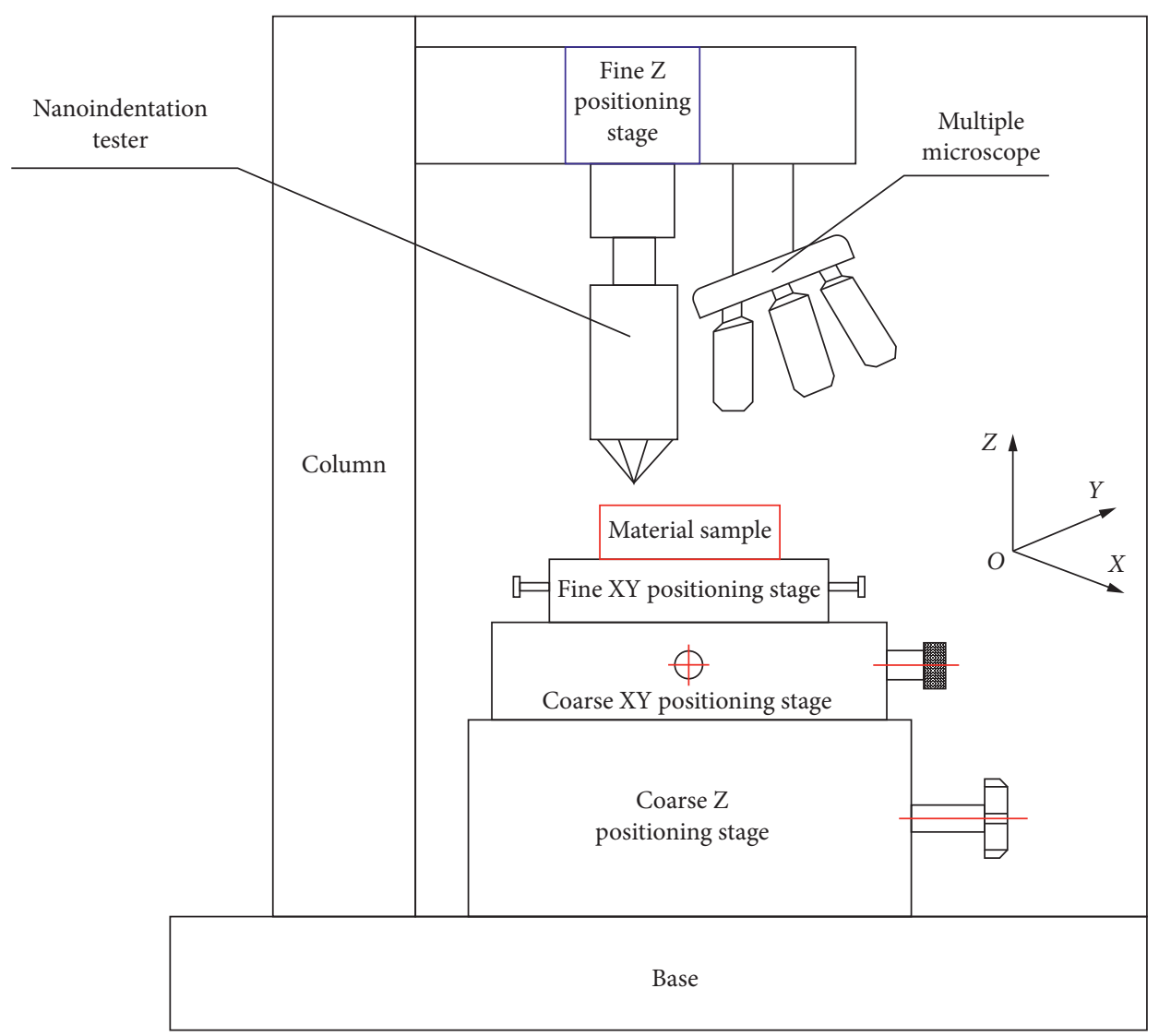

FIGURE 1: A proposed nano/microindentation system with the compliant z-stage.

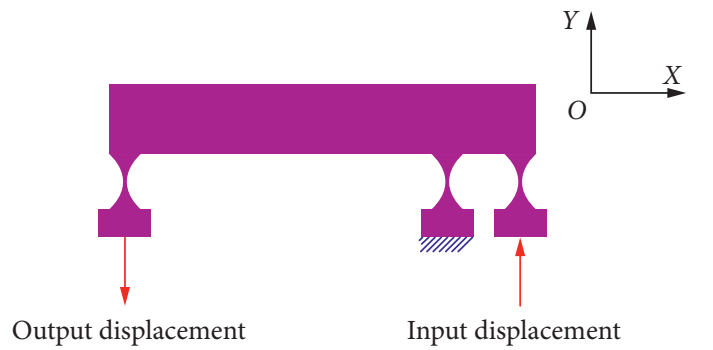

(a)

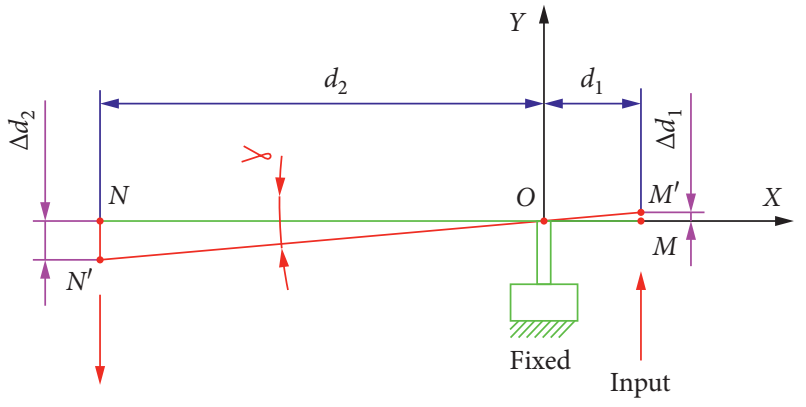

(b)

FIGURE 2: Scheme: (a) lever-structure operating; (b) magnification proportion.

Especially, based on the global convergence quality characteristics of the WOA, an integration approach of the TM, the RSM, weight factor calculation method based on signal to noise, and the whale optimization algorithm (WOA) is proposed for balancing among its characteristics as well as enhancing the quality responses of the $\mathrm{z}$-stage. A WOA algorithm would be exploited via MATLAB 2017. This integration method diagram is suggested, as shown in Figure 5.

The optimal trouble for the $z$-stage is shortened as follows.

Seek the input variables: $\mathbf{X}=[M, N, P, K]$.

Maximize $F_{1}(X)$ as follows:

$$
F_{1}(M, N, P, K) .
$$

Maximize $F_{2}(X)$ as follows:

$$
F_{2}(M, N, P, K),
$$

and subject to the following constraints:

$$
\sigma_{\max } \leq \frac{\sigma_{y}}{n},
$$

$\left\{\begin{array}{l}0.55 \mathrm{~mm} \leq M \leq 0.65 \mathrm{~mm} ; 0.6 \mathrm{~mm} \leq N \leq 0.9 \mathrm{~mm}, \\ 0.9 \mathrm{~mm} \leq P \leq 1.1 \mathrm{~mm} ; 49 \mathrm{~mm} \leq K \leq 53 \mathrm{~mm},\end{array}\right.$ 


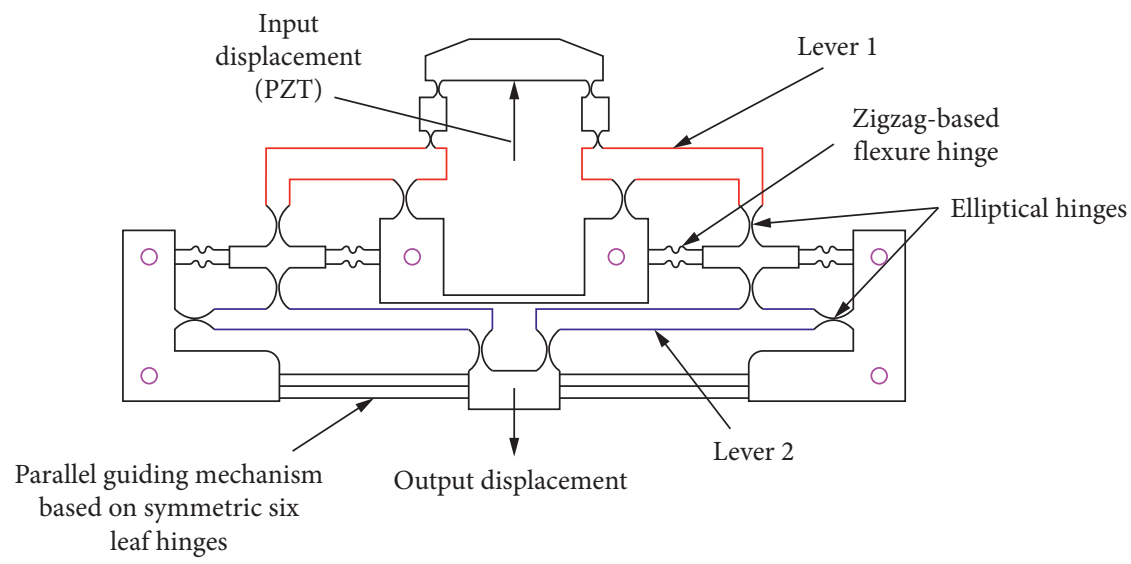

Figure 3: Proposed hybrid displacement amplifier.

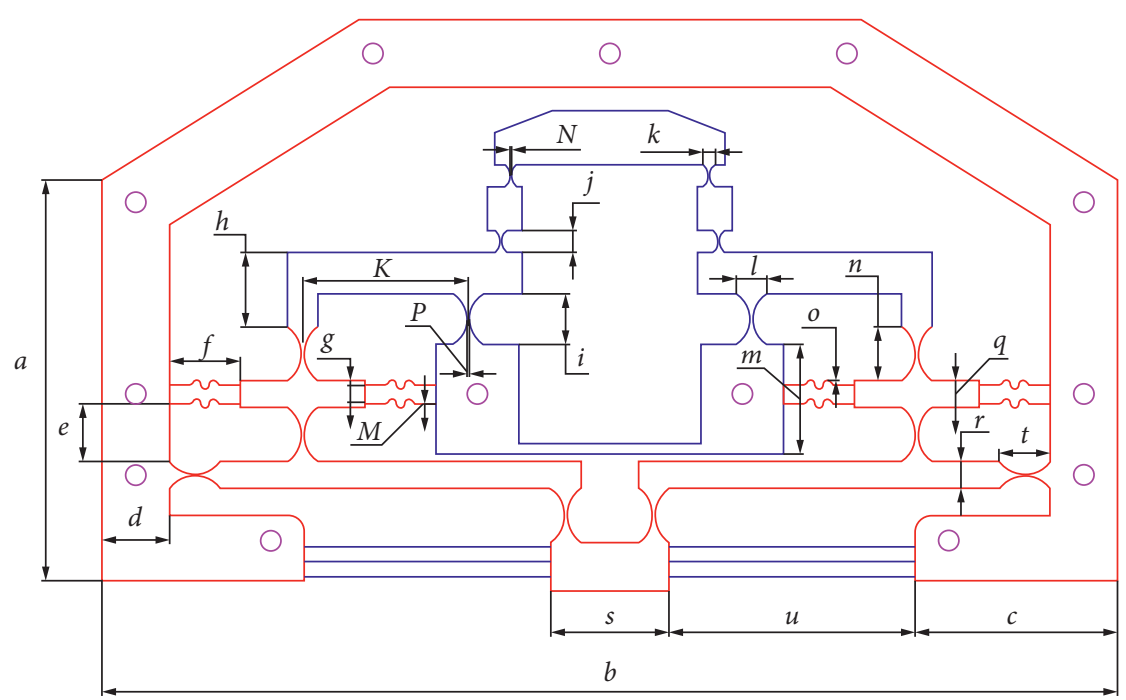

FIGURE 4: Main geometric factors of the z-stage.

TABLE 1: Geometric factors of the compliant z-stage.

\begin{tabular}{|c|c|c|c|c|c|c|}
\hline Factor & Worth & Factor & Worth & Factor & Worth & Unit \\
\hline $\bar{A}$ & 114.5 & $J$ & 6.5 & $i$ & 15 & $\mathrm{~mm}$ \\
\hline$B$ & 301 & K & 3.5 & $t$ & 15 & $\mathrm{~mm}$ \\
\hline C & 60 & $M$ & 32.5 & $u$ & 73 & $\mathrm{~mm}$ \\
\hline D & 20 & $N$ & 16 & $M$ & $0.55 \leq M \leq 0.65$ & $\mathrm{~mm}$ \\
\hline$E$ & 17 & $O$ & 1 & $N$ & $0.6 \leq N \leq 0.9$ & $\mathrm{~mm}$ \\
\hline$F$ & 21 & $Q$ & 8 & $P$ & $0.9 \leq P \leq 1.2$ & $\mathrm{~mm}$ \\
\hline$G$ & 5 & $R$ & 8 & K & $49 \leq K \leq 53$ & $\mathrm{~mm}$ \\
\hline$H$ & 22 & $S$ & 35 & & & \\
\hline
\end{tabular}

where $F_{1}$ and $F_{2}$ denote the safety factor and the displacement, respectively. There are many factors affecting to the output responses. Meanwhile, $M, N, P$, and $K$ are four main factors affecting significantly the output characteristics. Therefore, $M, N, P$, and $K$ were chosen as input design variables. For more specifics, $M, N, P$, and $K$ are thickness of zigzag-based compliant spring, thickness of first elliptical joint (lever-amplifier 1), thickness of second elliptical hinge (lever-amplifier 2), and distance between second elliptical hinge center and guiding elliptical hinge center, correspondingly.

Additionally, $\sigma_{y}$ symbolizes the yield strength of the material (Al 7075), $\sigma_{\max }$ symbolizes the maximal stress of the compliant stage as well as $n$ is the safety factor.

A safety factor of more than 1.8 was recommended to design $\mathrm{z}$-stage for avoiding plastic breakdowns. 


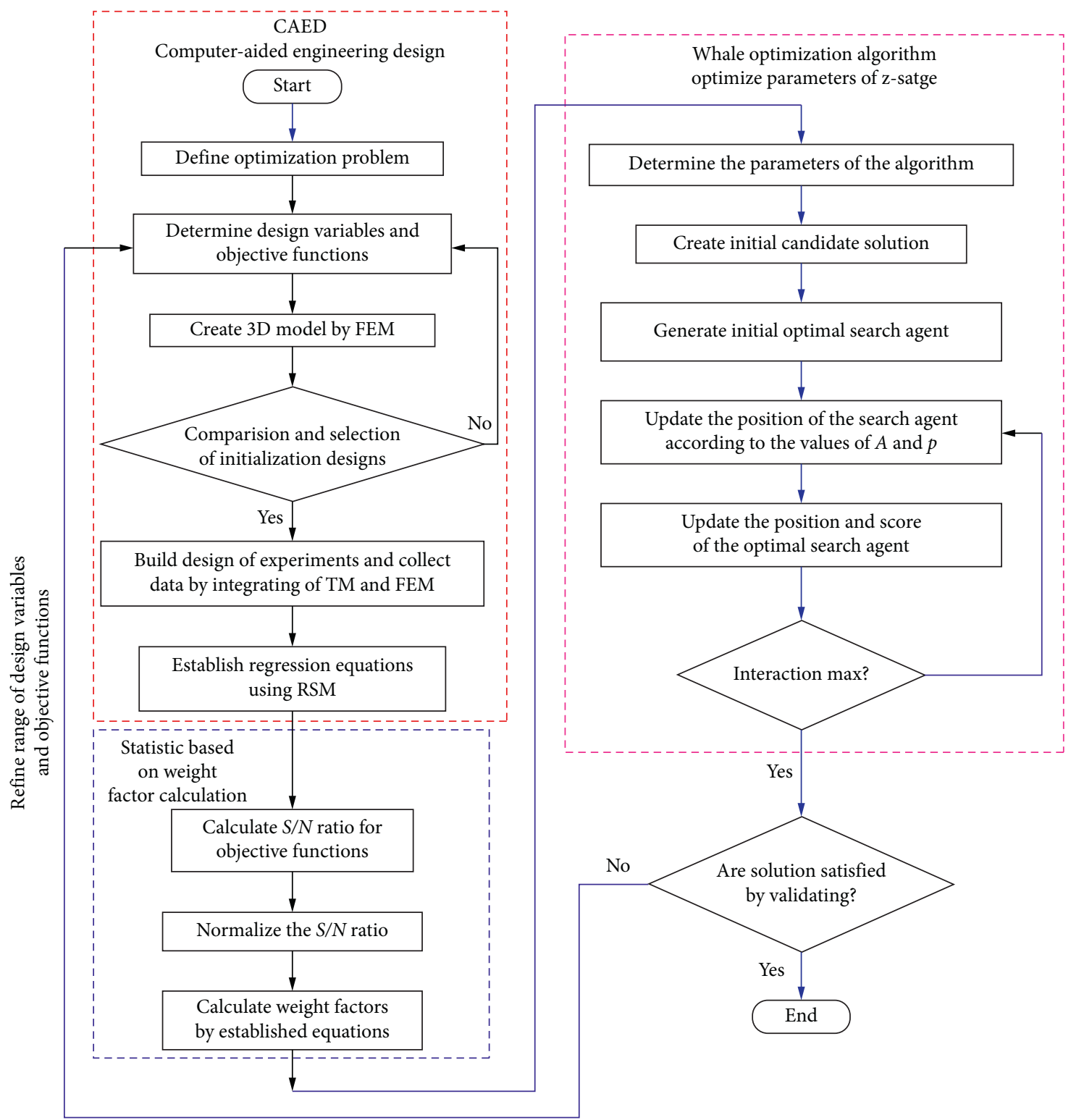

FIGURE 5: Flowchart of the proposed optimization approach.

Equation (6) illustrated limitation range of the input variables. The smaller and the higher bounds for the input variables were chosen for ensuring a compact compliant structure.

3.2. Hybrid Approach. In this research, the proposed method was developed to optimize the key geometric factors for enhancing the output responses of the stage.

Primarily, the TM is exploited for building initial numeric experiments. Secondly, the RSM is employed to form regression functions to map connections among input variables and the both quality characteristics. A complete quadratic formulation is appropriate type for the proposed z-stage as following equation:

$$
F_{j}=\beta_{0}+\sum_{i=1}^{n} \beta_{i} x_{i}+\sum_{i=1}^{n} \beta_{i j} x_{i}^{2}+\sum_{i=1}^{n-1} \sum_{j=i+1}^{n} \beta_{i j} x_{i} x_{j}+\varepsilon_{i},
$$

where $\beta_{i}(i=0,1,2, \ldots, n)$ denote unknown regression coefficients, $\beta_{i j}(i<j)$ denotes collaboration coefficients, $x_{1}$, $x_{2}, \ldots, x_{n}$ are series of $n$ predictors considered to be associated to a response variable $F_{j}$, and $\varepsilon$ denotes an arbitrary error.

Additionally, analysis of variance (ANOVA) would be implemented for defining meaningful influence proportion of every factors on the quality characteristics.

Thirdly, weight factors were determined for each response according to the signal to noise for enhancing optimal calculation results. Based on the weight factor method with details in Reference [53], the WFs for each response were computed accurately. Every objective is normalized in the variety from 0 to 1 . Later on, WFs were assigned for both output responses.

Based on the calculated weight factors, the total weighted objective function was conveyed as follows: 


$$
f=w_{1} f_{1}+w_{2} f_{2},
$$

where $f_{1}, f_{2}$, and $f$ are safety factor, output displacement, and integrated function, correspondingly and $w_{1}$ and $w_{2}$ denote the weight factor worths of $f_{1}$ and $f_{2}$, correspondingly. In addition, the weight factor is determined as follows:

(1) Identify Signal to Noise Scale $(S / N)$. The $S / N$ scale is formed by the Taguchi method, and it demonstrates a better ability corresponding to a higher signal to noise scale worth.

Based on the technical requirements of responses, the higher the better is exploited, that is, in form as follows:

$$
\eta=-10 \log \left(\frac{1}{k} \sum_{i=1}^{n} \frac{1}{y_{i}^{2}}\right),
$$

where $k$ and $y$ symbolize the number of reiterations of $i^{\text {th }}$ testing and the property.

(2) Normalize the $S / N$ Scale. The normalization-based signal/noise scale of every rank for every performance is expressed as follows:

$$
z_{i}=\frac{\eta_{j}-\min \eta_{j}}{\max \eta_{j}-\min \eta_{j}}
$$

where $\eta_{i}$ symbolizes the $S / N$ worth, $\max \eta_{i}$ and $\min \eta_{i}$ denote the greatest and lowest worths of $\eta_{i}$, respectively, $z_{i}$ symbolizes the normalization-based $S / N$ worth of characteristic function $(j=1,2, \ldots, k)$, and $k$ is the digit of characteristic functions.

(3) Define the Average Worth of Normalized S/N Scales. The average worth of normalized $S / N$ ratios at every grade for design variables was determined for characteristic functions. The $S / N$ scale worths are normalized, and the average worth of normalized $S /$ $N$ scales is in form as follows:

$$
a_{L i}=\frac{1}{N_{L i}} \sum_{i}^{m} z_{i j}
$$

where $N_{L j i}$ symbolizes digit of reiteration of grade $i$ th, $z_{i j}$ symbolizes the worth of $S / N$ scale of grade $i$ th of performance function $j$ th, and $A l_{i}$ is the mean value of $S / N$ ratio of grade $i$ th of every input variable of every performance function.

(4) Determine the Variety of Every Design Variable. The variety of the every grade of every design variable is expressed as follows:

$$
\begin{aligned}
r_{i j}= & \max \left\{z_{i, j, 1}, z_{i, j, 2}, \ldots, z_{i, j, m}\right\} \\
& -\min \left\{z_{i, j, 1}, z_{i, j, 2}, \ldots, z_{i, j, m}\right\},
\end{aligned}
$$

where $r_{i j}$ denotes the variety (max-min) of the $S / N$ value for every grade of every parameter; $j=1,2, \ldots, q$, whereq is the digit of input variables; and $m=1,2, \ldots, l$, where $l$ denotes the digit of experiment grade of every input variable.

(5) Identify the Weight Factors (WFs). The WFs are in form as follows:

$$
w_{i}=\frac{\sum_{j=1}^{q} r_{i j}}{\sum_{i=1}^{m_{O F}} \sum_{j=1}^{q} r_{i j}},
$$

where $w_{i} \quad\left(w_{i} \geq 0\right)$ symbolizes the WF of every performance function ith and $m_{O F}$ symbolizes the digit of performance functions.

The equation for checking the calculation the sum of weight factors is given by

$$
\sum_{i=1}^{m_{\text {OF }}} w_{i}=1
$$

According to the regression functions assigned calculated weight factors, the whale optimization algorithm was employed for seeking the optimization factors. A WOA was proposed for multiple responses optimization because of the quality convergence characteristics and high convergence speed.

3.3. Whale Optimization Algorithm. Finally, the WOA imitated from hunting manners of the humpback whales in seeking and tackling preys considering bubble-net feeding manner was suggested by Mirjalili and Lewis [18]. The WOA comprises two key phases as follows: (1) exploitation phase: surrounding quarry and helix apprising location can be executed and (2) Exploration phase: investigating for a prey is conducted erratically. The more details can be read in Reference [18]. The mathematical equation of every phase is expressed in the subsequent parts.

3.3.1. Exploitation Phase (Bubble-Net Attacking Method). Both progresses can be established so as to establish mathematical function for the bubble-net manner of humpback whales. The both progresses are expressed as follows.

(1) Surrounding Quarry. After identifying the location of the quarry, humpback whales surround the quarry. Because the situation of the optimization design in the investigation area can be not defined, the WOA algorithm supposes that the recent finest applicant resolution is the objective quarry or is proximate the optimum. Later on, the greatest investigation agent is determined. Therefore, the other investigation agents can endeavor for adapting their situations nearby the finest investigation agent. Mathematical equations of the manner are in form as follows:

$$
\begin{aligned}
\vec{D} & =\left|\vec{C} \cdot \overrightarrow{X^{*}}(t)-\vec{X}(t)\right|, \\
\vec{X}(t+1) & =\overrightarrow{X^{*}}(t)-\vec{A} \cdot \vec{D},
\end{aligned}
$$


where $t$ denotes the present repetition, $X^{*}$ symbolizes the location vector of the greatest gained resolution, $X$ symbolizes the location vector, and || is the absolute value. $X^{*}$ ought to be apprised in every iteration if it has a greater resolution. $A$ and $C$ are coefficient vectors computed and are in form as follows:

$$
\begin{aligned}
& \vec{A}=2 \vec{a} \cdot \vec{r}-\vec{a}, \\
& \vec{C}=2 \cdot \vec{r},
\end{aligned}
$$

where $\vec{a}$ is declined in range $(2,0)$ throughout repetitions and $\vec{r}$ symbolizes an arbitrary vector in range $(0,1)$.

To employ shrinking, the worth of $\vec{a}$ is decreased in equation (17). In addition, the oscillation variety of $\vec{A}$ can be reduced via $\vec{a}$. Besides, $\vec{A}$ symbolizes an arbitrary worth in the interval from-a to a where a is declined in $(2,0)$ over the course of repetitions. Establishing arbitrary worths for $\vec{A}$ from -1 to 1 , the updated location of an investigation agent is specified anywhere from the initial point of the agent to the location of the recent greatest agent.

3.4. Helix Updating Location. After computing the dimension among the whale and quarry corresponding with positions at $(X, Y)$ and $\left(X^{*}, Y^{*}\right)$, Later on, a spiral model for the place of whale and prey to imitate the spiral shape-based motion of humpback whales is computed as the following equation:

$$
\vec{X}(t+1)=\overrightarrow{D^{\prime}} \cdot e^{b m} \cdot \cos (2 \pi l)+\overrightarrow{X^{*}}(t),
$$

where $\overrightarrow{D^{\prime}}=\left|\overrightarrow{X^{*}}(t)-\vec{X}(t)\right|$ and shows the distance of the $i$ th whale to the quarry, $b$ symbolizes a stable worth for determining the form of the logarithmic helix, and $m$ symbolizes an arbitrary digit form -1 to 1 . This manner in WOA is illustrated for changing locations of whales in optimal process. To formulate simultaneously this manner, it has a $50 \%$ possibility for preferring amongst the shrinking encircling procedure and the helix pattern. The mathematical model for updated location of whales is calculated as following equation:

$$
\vec{X}(t+1)=\left\{\begin{array}{cc}
\overrightarrow{X^{*}}(t)-\vec{A} \cdot \vec{D} & \text { if } p<0.5 \\
\overrightarrow{D^{\prime}} \cdot e^{b l} \cdot \cos (2 \pi l)+\overrightarrow{X^{*}}(t) & \text { if } p<0.5
\end{array}\right\},
$$

where $p$ denotes the contingent value from 0 to 1 .

3.5. Exploration Phase (Investigation for Quarry). The similar method according to the difference of the $\vec{A}$ vector can be exploited for investigating for quarry (exploration). Actually, humpback whales are investigated arbitrarily according to the place of every another. Consequently, $\vec{A}$ can be utilized as the arbitrary worths better than 1 or less than -1 to control investigation agent to journey remotely with a reference whale. Until now, compared with the exploitation phase, the place of an investigation agent in the exploration phase is updated based on an arbitrarily chosen investigation agent in preference to the greatest investigation agent realized. This structure and $|\vec{A}|>1$ highlight exploration as well as permit the WOA algorithm to implement a universal investigation. The algebraic equation can be computed by

$$
\begin{aligned}
\vec{D} & =\left|\vec{C} \cdot X_{\text {rand }}-\vec{X}\right|, \\
\vec{X}(t+1) & =X_{\text {rand }}^{-}-\vec{A} \cdot \vec{D},
\end{aligned}
$$

where $X_{\text {rand }}^{-}$symbolizes an arbitrary location vector (an arbitrary whale) preferred according to present population.

3.6. Process of Whale Optimization Algorithm. The WOA algorithm starts via setting arbitrary resolutions. Until now, investigation agents apprise their places with respect to either an arbitrarily selected investigation agent or the greatest resolution gained for every repetition. The factor is fallen in range $(2,0)$ so as to offer exploration and exploitation, correspondingly. An arbitrary investigation agent is preferred when $|\vec{A}|>1$, while the greatest resolution is chosen when $|\vec{A}|<1$ for apprising the location of the investigation agents. Therefore, if its value is more than 0.5 , then the investigation agents vary their points using equation (19), else utilizing equation (16). WOA is able to exchange among either a helix or rounded travel. Ultimately, the WOA algorithm is ended by the fulfilment of an ending condition.

Based on theoretical view, the WOA can be concerned about a universal optimization algorithm according to its merits of exploration/exploitation ability. Moreover, the proposed hypercube structure determines an investigation region in the area of the best resolution and permits other investigation agents to utilize the current greatest evidence in the domain. Apprised alteration of the search vector $A$ permits the WOA algorithm to smooth journey among exploration as well as exploitation: by $\operatorname{declining} A$, several repetitions can be dedicated to exploration $(|A| \geq 1)$, and the rest is allocated to exploitation $(|A|<1)$. Extraordinarily, the proposed algorithm comprises purely both key internal factors modified ( $A$ and $C$ ). The more details can be read in Reference [18].

\section{Results and Discussion}

4.1. Assessment of Primary Characteristics and Decoupling Error. Firstly, a compliant $\mathrm{z}$-stage was constructed via Inventor software. Subsequently, the $z$-axis output displacement as well as safety factor of the proposed z-stage was retrieved based on FEA in Ansys software. For specifics, a coarse meshing was employed in the automatic mode. Thereafter, compliant joints were purified for achieving accurate analysis results, as demonstrated in Figure 6.

In order to check the safety factor, the decoupling error, and magnification proportion, as illustrated in Table 2, the output deformation of $\mathrm{z}$-stage was examined via altering the input displacement from $5 \mu \mathrm{m}$ to $60 \mu \mathrm{m}$, correspondingly. 


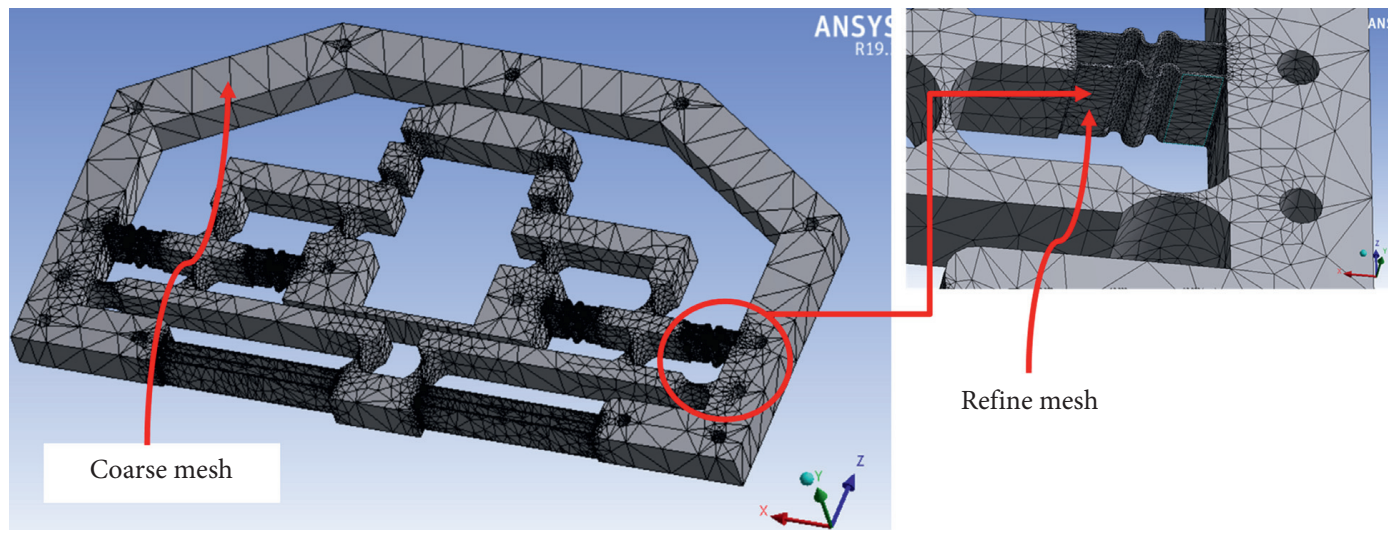

Figure 6: Mesh establishment of the proposed z-stage.

TABLE 2: The output deformation, magnification, safety factor, and stress.

\begin{tabular}{|c|c|c|c|c|}
\hline Input displacement $(\mu \mathrm{m})$ & Output deformation $(\mu \mathrm{m})$ & Magnification scale & Safety factor & $\begin{array}{c}\text { Equivalent stress } \\
(\mathrm{MPa})\end{array}$ \\
\hline 65 & 387 & 5.95 & 1.8092 & 278.02 \\
\hline 70 & 416.77 & 5.95 & 1.68 & 299.41 \\
\hline 75 & 446.54 & 5.95 & 1.568 & 320.8 \\
\hline 80 & 476.31 & 5.95 & 1.47 & 342.18 \\
\hline 85 & 506.07 & 5.95 & 1.3835 & 363.57 \\
\hline 90 & 535.84 & 5.95 & 1.3066 & 384.96 \\
\hline 95 & 565.61 & 5.95 & 1.2379 & 406.34 \\
\hline 100 & 595.38 & 5.95 & 1.176 & 427.73 \\
\hline 105 & 625.15 & 5.95 & 1.12 & 449.12 \\
\hline 110 & 654.92 & 5.95 & 1.0691 & 470.5 \\
\hline 115 & 684.69 & 5.95 & 1.0226 & 491.89 \\
\hline 120 & 714.46 & 5.95 & 0.97998 & 513.28 \\
\hline
\end{tabular}

In order to check strength conditions of the proposed $\mathrm{Z}$-stage, the input movement was changed in range $(65,120)$ with the changing step of $5 \mu \mathrm{m}$. In addition, the corresponding output deformation could be retrieved by FEA. Based on the consequences of Table 2, corresponding to input displacement of $120 \mu \mathrm{m}$, the output deformation was $714.46 \mu \mathrm{m}$, the safety factor was lower than 1 as well as the stage could be damaged. Consequently, the maximal input displacement ought to be lower than $120 \mu \mathrm{m}$. By contrast, when the input displacement was $115 \mu \mathrm{m}$, the equivalent stress was around $491.89 \mathrm{MPa}$ (lower than the yield strength of material) corresponding to the safety factor of 1.0226. Especially, in order to ensure a good working life for the stage, a safety factor over 1.8 was expected. In addition, the input displacement was proposed range in (5, 65 ) for checking output deformation as well as the decoupling error which was computed via exploiting equation (21). The consequences showed that the decoupling error was moderately minor with $0.0389 \%(<1 \%)$, as shown in Table 3 . Therefore, it guaranteed the accuracy for the proposed $z$-stage. Moreover, the consequences exhibited the safety factor of 1.8092 and magnification scale (MS) of 5.95. Conversely, all consequences could not meet magnification demands (MS is more than 6). Consequently, an optimal procedure should be implemented for enhancing the quality characteristics.
When the $\mathrm{z}$-stage travelled along the $z$-direction, similarly, the motion of $x$-direction also occurred. The unexpected travel created the decoupling error. Based on the results of Table 3 , the decoupling error is really minor with $0.0389 \%(<1 \%)$, so, this error did not influence meaningfully to the output deformation accuracy. The decoupling error could be in form as follows:

$$
e_{d}=\frac{x_{o}}{z_{o}} 100 \%
$$

where $e_{d}$ symbolizes the decoupling error and $x_{o}$ and $z_{o}$ are the $x$-direction output deformation and the $z$-direction output deformation.

4.2. Orthogonal Array Experiment and Mathematical Model. Every parameter could be partitioned to triple grades according to specialized experience as well as machining capacity, as revealed in Table 4 . The $L_{9}\left(3^{4}\right)$ orthogonal array of the Taguchi method was implemented for forming the initial quantity of experimentations. The safety factor $\left(F_{1}\right)$ and $z$ probed deformation $\left(F_{2}\right)$ were gathered by the finite element method in Ansys software and consequences of the numeric testing, as exhibited in Table 5. 
TABLE 3: Consequences of output deformation as well as decoupling error.

\begin{tabular}{|c|c|c|c|c|}
\hline Input displacement $(\mu \mathrm{m})$ & $Z$-axis output deformation $(\mu \mathrm{m})$ & $X$-axis output deformation $(\mu \mathrm{m})$ & Decoupling error $(\%)$ & Magnification scale \\
\hline 5 & 29.77 & 0.0116 & 0.0389 & 5.95 \\
\hline 10 & 59.54 & 0.0232 & 0.0389 & 5.95 \\
\hline 15 & 89.31 & 0.0348 & 0.0389 & 5.95 \\
\hline 20 & 119.08 & 0.0464 & 0.0389 & 5.95 \\
\hline 25 & 148.85 & 0.0579 & 0.0389 & 5.95 \\
\hline 30 & 178.61 & 0.0695 & 0.0389 & 5.95 \\
\hline 35 & 208.38 & 0.0811 & 0.0389 & 5.95 \\
\hline 40 & 238.15 & 0.0927 & 0.0389 & 5.95 \\
\hline 45 & 267.92 & 0.1043 & 0.0389 & 5.95 \\
\hline 50 & 297.69 & 0.1159 & 0.0389 & 5.95 \\
\hline 55 & 327.46 & 0.1275 & 0.0389 & 5.95 \\
\hline 60 & 357.23 & 0.1391 & 0.0389 & 5.95 \\
\hline
\end{tabular}

TABLE 4: Input dimensions and their grades (unit: $\mathrm{mm}$ ).

\begin{tabular}{lcccc}
\hline Parameters & Variety & Grade 1 & Grade 2 & Grade 3 \\
\hline$M$ & $0.55-0.65$ & 0.55 & 0.6 & 0.65 \\
$N$ & $0.6-0.9$ & 0.6 & 0.75 & 0.9 \\
$P$ & $0.9-1.2$ & 0.9 & 1.05 & 1.2 \\
$K$ & $49-53$ & 49 & 51 & 53 \\
\hline
\end{tabular}

TABLe 5: Numerical results.

\begin{tabular}{|c|c|c|c|c|c|c|}
\hline No. & $M$ & $N$ & $P$ & $K$ & $F_{1}$ & $F_{2}(\mu \mathrm{m})$ \\
\hline 1 & 0.55 & 0.6 & 0.9 & 49 & 2.1846 & 423.89 \\
\hline 2 & 0.55 & 0.75 & 1.05 & 51 & 1.8968 & 392.88 \\
\hline 3 & 0.55 & 0.9 & 1.2 & 53 & 1.8956 & 253.77 \\
\hline 4 & 0.6 & 0.6 & 1.05 & 53 & 1.615 & 221.14 \\
\hline 5 & 0.6 & 0.75 & 1.2 & 49 & 2.1052 & 401.83 \\
\hline 6 & 0.6 & 0.9 & 0.9 & 51 & 1.944 & 370.74 \\
\hline 7 & 0.65 & 0.6 & 1.2 & 51 & 1.6641 & 309.1 \\
\hline 8 & 0.65 & 0.75 & 0.9 & 53 & 1.7837 & 211.66 \\
\hline 9 & 0.65 & 0.9 & 1.05 & 49 & 2.2807 & 381.09 \\
\hline
\end{tabular}

The regression equations were gained as follows:

$$
\begin{aligned}
F_{1}= & 109.2-31.00^{*} M+0.5896^{*} N-0.1536^{*} P-3.740^{*} K+25.14^{*} M^{*} M \\
& +0.09333^{*} N^{*} N-0.05778^{*} P^{*} P+0.03562^{*} K^{*} K, \\
F_{2}= & -25134+640.1^{*} M+646.2^{*} N+252.9^{*} P+1028^{*} K-1002^{*} M^{*} M \\
& -392.7^{*} N^{*} N-142.4^{*} P^{*} P-10.50^{*} K^{*} K .
\end{aligned}
$$

Tables 6 and 7 show the ANOVA consequences of the safety factor as well as output deformation. The assessment could be implemented with 5\% substance grade and $95 \%$ assurance grade.

As depicted in Table 6, the impact rate on the safety factor $F_{1}$ of $K$ was highest, at $84.34 \%$, while the effect figure for $N$ and interaction $K$ and $K$ was substantial higher, at $17.43 \%$ and $9.85 \%$, respectively. In contrast, the rate of $M, P$ as well as interaction $M$ and $M$ was slightly lower, at $2.50 \%$, $2.47 \%$, and $1.92 \%$, respectively. Consequently, so as to climb the worth of $F_{1}$, factors $K$ as well as $N$ ought to be considerably monitored.

As illustrated in Table 7 , the effect rate of $K$ on $F_{2}$ was greatest, at $83.07 \%$, while the effect figure for $M$ and relation $K$ and $K$ was substantial higher, at $8.73 \%$ and $6.50 \%$, respectively. In contrast, the rate of $N, P$, relation $N$ and $N$, relation $P$ and $P$ as well as relation $M$ and $M$ was slightly lower, at $0.81 \%, 0.53 \%, 0.29 \%, 0.04 \%$, and $0.02 \%$, respectively. Hence, so as to climb the worth of $F_{1}$, parameters $K$ and $M$ ought to be considerably monitored. Furthermore, the influence rate of error for $F_{1}$ as well as $F_{2}$ was $0 \%$.

4.3. Sensitivity Analysis. Statistic engineering was implemented to define the influence level of input variables on the output performances. In the variety $(0.55,0.65)$, factor $M$ influenced to $F_{1}$ and $F_{2}$ in reducing slightly, as depicted in 
TABLE 6: ANOVA analysis for safety factor $\left(F_{1}\right)$.

\begin{tabular}{|c|c|c|c|c|c|c|}
\hline Source & $\mathrm{DF}$ & Seq SS & Contribution (\%) & Adj SS & Adj MS & $P$ value \\
\hline Model & 8 & 0.412320 & 100.00 & 0.412320 & 0.051540 & Substantial \\
\hline Linear & 4 & 0.363795 & 88.23 & 0.363795 & 0.090949 & Substantial \\
\hline$M$ & 1 & 0.010292 & 2.50 & 0.010292 & 0.010292 & Substantial \\
\hline$N$ & 1 & 0.071854 & 17.43 & 0.071854 & 0.071854 & Substantial \\
\hline$P$ & 1 & 0.010201 & 2.47 & 0.010201 & 0.010201 & Substantial \\
\hline K & 1 & 0.271448 & 65.83 & 0.271448 & 0.271448 & Substantial \\
\hline Square & 4 & 0.048525 & 11.77 & 0.048525 & 0.012131 & Substantial \\
\hline$M * M$ & 1 & 0.007900 & 1.92 & 0.007900 & 0.007900 & Substantial \\
\hline$N * N$ & 1 & 0.000009 & 0.00 & 0.000009 & 0.000009 & Substantial \\
\hline$P * P$ & 1 & 0.000003 & 0.00 & 0.000003 & 0.000003 & Substantial \\
\hline$K * K$ & 1 & 0.040613 & 9.85 & 0.040613 & 0.040613 & Substantial \\
\hline Error & 0 & - & - & - & - & - \\
\hline Total & 8 & 0.412320 & 100.00 & 一 & - & - \\
\hline
\end{tabular}

TABLe 7: ANOVA analysis for output displacement $\left(F_{2}\right)$.

\begin{tabular}{|c|c|c|c|c|c|c|}
\hline Source & DF & Seq SS & Contribution (\%) & Adj SS & Adj MS & $P$ value \\
\hline Model & 8 & 54299.7 & 100.00 & 54299.7 & 6787.5 & Substantial \\
\hline Linear & 4 & 50580.8 & 93.15 & 50580.8 & 12645.2 & Substantial \\
\hline$M$ & 1 & 4742.7 & 8.73 & 4742.7 & 4742.7 & Substantial \\
\hline$N$ & 1 & 441.5 & 0.81 & 441.5 & 441.5 & Substantial \\
\hline$P$ & 1 & 288.3 & 0.53 & 288.3 & 288.3 & Substantial \\
\hline$K$ & 1 & 45108.3 & 83.07 & 45108.3 & 45108.3 & Substantial \\
\hline Square & 4 & 3718.9 & 6.85 & 3718.9 & 929.7 & Substantial \\
\hline$M^{*} M$ & 1 & 12.6 & 0.02 & 12.6 & 12.6 & Substantial \\
\hline$N^{*} N$ & 1 & 156.1 & 0.29 & 156.1 & 156.1 & Substantial \\
\hline$P^{*} P$ & 1 & 20.5 & 0.04 & 20.5 & 20.5 & Substantial \\
\hline$K^{*} K$ & 1 & 3529.7 & 6.50 & 3529.7 & 3529.7 & Substantial \\
\hline Error & 0 & - & - & - & - & - \\
\hline Total & 8 & 54299.7 & 100.00 & - & - & - \\
\hline
\end{tabular}

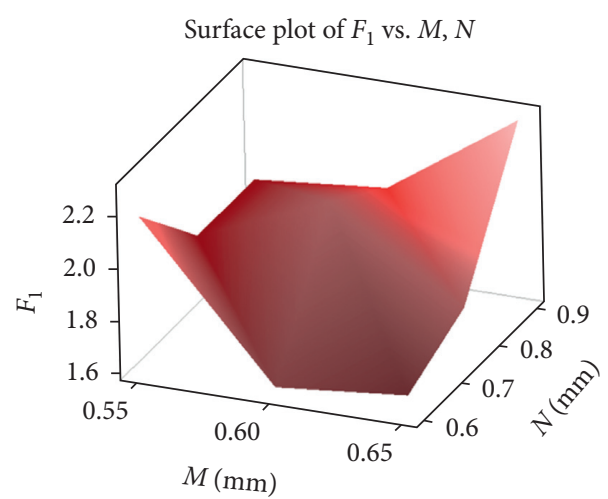

(a)

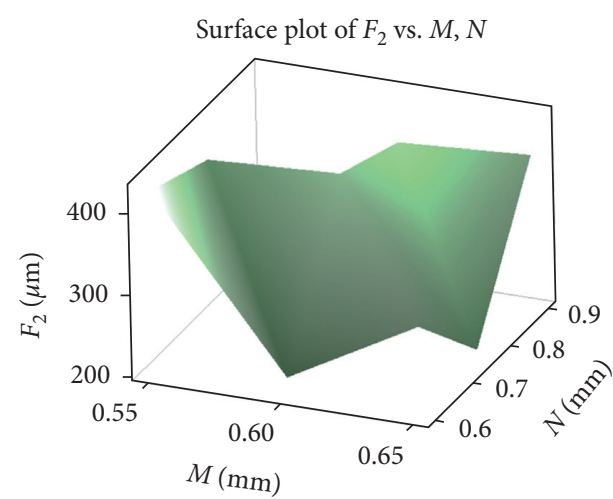

(b)

Figure 7: Influence scheme of $M$ and $N$ on (a) safety factor and (b) the output deformation.

Figure 7. By contrast, element $N$ revealed that in the variety in $(0.65,0.75)$, it influenced a gradual increase to $F_{1}$ as well as affected a slow fluctuation to $F_{2}$.

In the variety $(0.9,1.2)$, factor $P$ influenced a slow decline to $F_{1}$ and $F_{2}$, as depicted in Figure 8 . In addition, in the variety $(50,51.5)$, factor $K$ influenced a sharp decline to $F_{1}$ and affected a gradual decline to $F_{2}$. Meanwhile, from $51.5 \mathrm{~mm}$ to $53 \mathrm{~mm}$, factor $K$ influenced a slight decline to $F_{1}$ as well as caused a dramatic decline to $F_{2}$.
To sum up, total influences of input variables on output responses were demonstrated, as in Figure 9. These depict a rise and decline variety in every parameter. Based on these results, the main factors could be adjusted for obtaining a greatest construction for the $\mathrm{z}$-stage.

4.4. Calculation of Weight Factor. In general, total of both weight factors were equivalent to one. In addition, this 


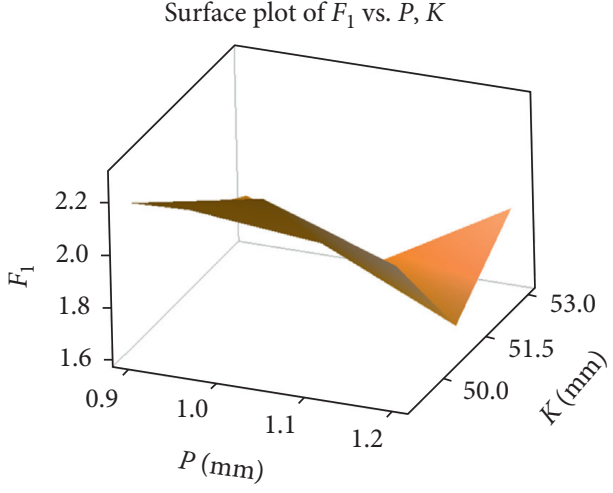

(a)

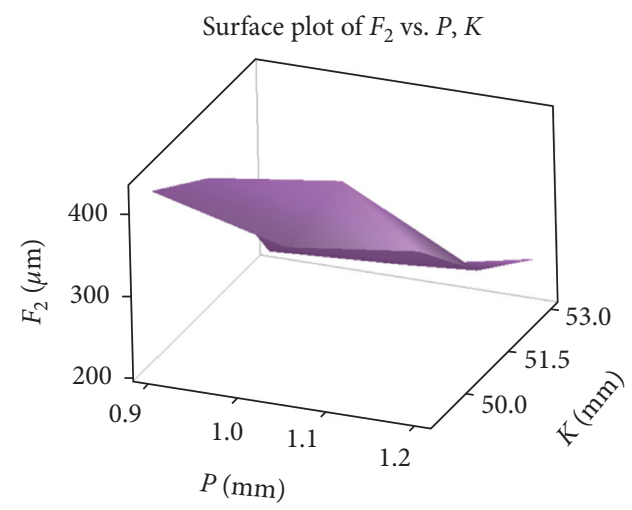

(b)

Figure 8: Influence scheme of $P$ and $K$ on (a) safety factor and (b) the output deformation.

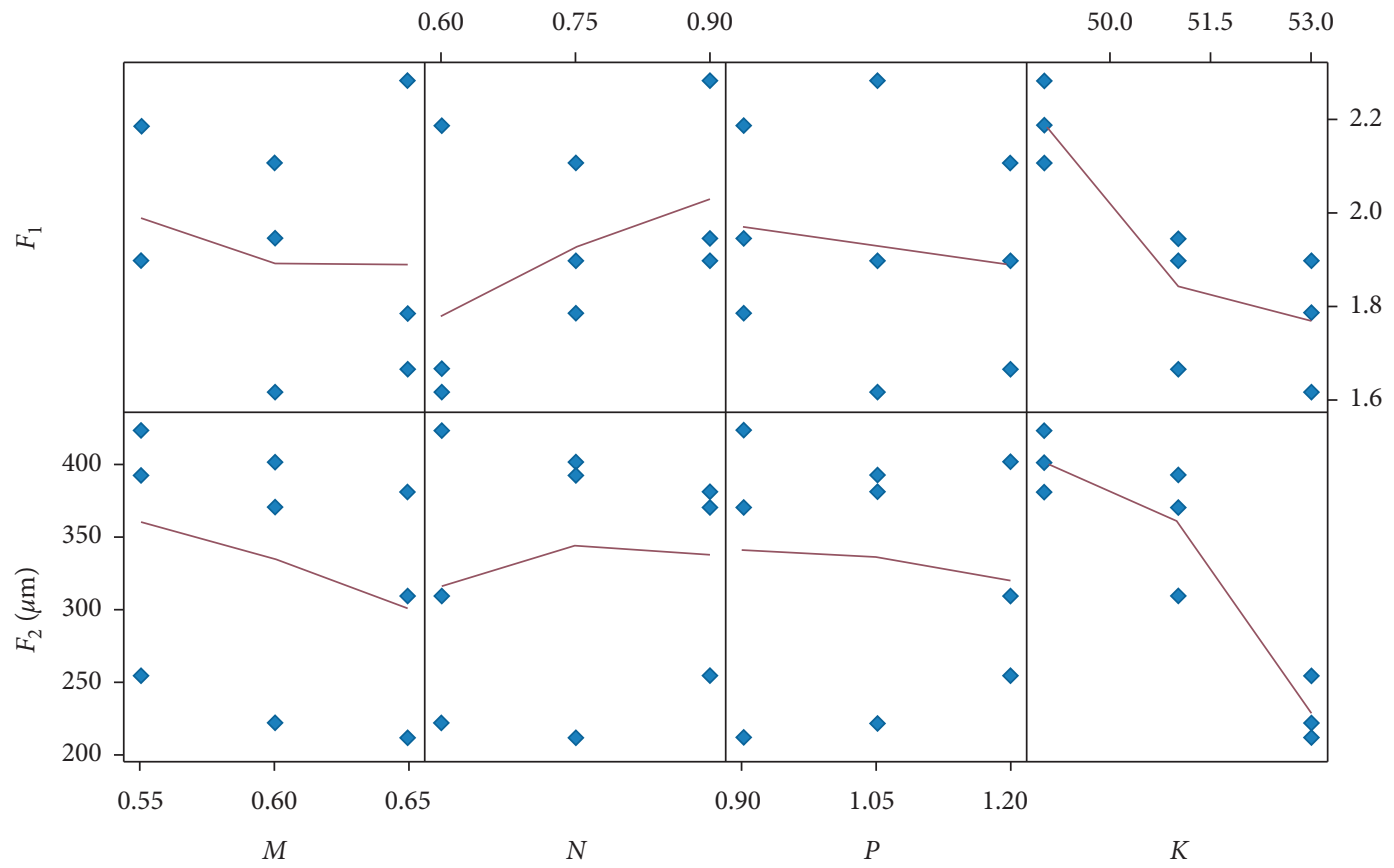

FIgURE 9: Sensibility scheme of input variables on two quality characteristics.

weight factor for every response was allocated 0.5 but this worth could be not exact as well as found optimal solutions could be imprecise. Consequently, this paper proposed to exploit an efficient method to define the weight factors.

Afterward, the numerical data were transferred into the signal to noise proportions applying equation (9), as revealed in Table 8 . Subsequently, the proportions were normalized via equation (10), as demonstrated in Table 9. For specifics, the normalized signal to noise proportions for safety factor $\left(\eta_{1}\right)$ and the displacement $\left(\eta_{2}\right)$ symbolized $z_{1}$ and $z_{2}$, correspondingly. Employing equations (12) and (13), the WF for the safety factor and the displacement was computed, as indicated in Tables 10 and 11, correspondingly. The weight factor worths for $F_{1}$ and $F_{2}$ were 0.5150 and 0.485 , correspondingly. Later on, the calculated WFs for each response were allocated for the optimal procedure in the WOA.
4.5. Optimal Results and Verifications. Initial numeric experimentations were generated via exploiting the Taguchi method. Subsequently, the $\mathrm{z}$-stage design was constructed and the numeric calculation results were retrieved. According to collected information, the response surface method was employed for forming the regression models for the safety factor as well as output deformation. Afterward, the WFs were determined using equations (9)-(14). According to the weight factors assigned in equation (8), the multiobjective optimization trouble was resolved via the WOA method. The optimal procedure was executed by utilizing MATLAB 2017 software. The optimization consequences were detected at $M=0.55 \mathrm{~mm}$, $N=0.82377 \mathrm{~mm}, P=0.9 \mathrm{~mm}, K=49 \mathrm{~mm}, F_{1}=2.382456817$ as well as $F_{2}=454.5511266 \mu \mathrm{m}$. The consequences exposed that the optimal values of both response were appropriate for the mirco/nanoindentation device and precise positioning system as well as assured strength conditions of proposed material. 
TABLE 8: The investigational consequences and $S / N$ proportions.

\begin{tabular}{lcccc}
\hline No. & $F_{1}$ & $F_{2}(\mathrm{~mm})$ & $\eta_{1}$ of $F_{1}(\mathrm{~dB})$ & $\eta_{2}$ of $F_{2}(\mathrm{~dB})$ \\
\hline 1 & 2.1846 & 423.89 & 6.787438586 & 52.54506342 \\
2 & 1.8968 & 392.88 & 5.560430819 & 51.88519842 \\
3 & 1.8956 & 253.77 & 5.554934001 & 48.088805559 \\
4 & 1.615 & 221.14 & 4.163450533 & 46.89334611 \\
5 & 2.1052 & 401.83 & 6.465867227 & 52.08084715 \\
6 & 1.944 & 370.74 & 5.773925212 & 51.38138891 \\
7 & 1.6641 & 309.1 & 4.423588412 & 49.8019801 \\
8 & 1.7837 & 211.66 & 5.026436246 & 46.51277584 \\
9 & 2.2807 & 381.09 & 7.161363251 & 51.62055106 \\
\hline
\end{tabular}

TABLE 9: The worths of normalized $S / N$ proportions $\left(z_{i}\right)$.

\begin{tabular}{lccr}
\hline & $S / N$ proportions & \multicolumn{2}{c}{ Normalized $S / N$ proportions $\left(z_{i}\right)$} \\
$\eta_{1}(\mathrm{~dB})$ & $\eta_{2}(\mathrm{~dB})$ & $z_{1}$ of $\eta_{1}$ & 1.0000 \\
\hline 6.787438586 & 52.54506342 & 0.8753 & 0.8906 \\
5.560430819 & 51.88519842 & 0.4660 & 0.2613 \\
5.554934001 & 48.08880559 & 0.4642 & 0.0631 \\
4.163450533 & 46.89334611 & 0.0000 & 0.9230 \\
6.465867227 & 52.08084715 & 0.7680 & 0.8071 \\
5.773925212 & 51.38138891 & 0.5372 & 0.5453 \\
4.423588412 & 49.8019801 & 0.0868 & 0.0000 \\
5.026436246 & 46.51277584 & 0.2879 & 0.8467 \\
7.161363251 & 51.62055106 & 1.0000 & \\
\hline
\end{tabular}

TABLe 10: The WF for $F_{1}$.

\begin{tabular}{lcccc}
\hline Grade & \multicolumn{4}{c}{ The average worth of normalized $S / N$ scales at } \\
& $M$ & $N$ & $P$ & $K$ \\
\hline Grade 1 & 0.6018 & 0.3207 & 0.5668 & 0.8811 \\
Grade 2 & 0.4351 & 0.5073 & 0.4887 & 0.3633 \\
Grade 3 & 0.4582 & 0.6671 & 0.4396 & 0.2507 \\
Variety $r_{i j}$ & 0.1667 & 0.3464 & 0.1271 & 0.6304 \\
\multicolumn{5}{c}{ WF for $\mathbf{F}_{\mathbf{1}}: \mathbf{w}_{\mathbf{1}}=\mathbf{0 . 5 1 5 0}$} \\
\hline
\end{tabular}

TABle 11: The WF for $F_{2}$.

\begin{tabular}{lcccc}
\hline Grade & \multicolumn{4}{c}{ The average worth of normalized $S / N$} \\
& $M$ & $N$ & $P$ & $K$ \\
\hline proportions at every grade \\
Grade 1 & 0.7173 & 0.5361 & 0.6024 & 0.9233 \\
Grade 3 & 0.5977 & 0.6046 & 0.6001 & 0.7477 \\
Variety $r_{i j}$ & 0.4640 & 0.6384 & 0.5765 & 0.1081 \\
\multicolumn{5}{c}{ WF for $\mathbf{F}_{\mathbf{2}}: \mathbf{w}_{\mathbf{2}}=\mathbf{0 . 4 8 5}$} \\
\hline
\end{tabular}

4.5.1. Dynamic Analysis. These optimal parameters of $\mathrm{Z}$-stage built $3 \mathrm{D}$ model again in order to check dynamic characteristics for six modes of natural resonant frequency. The modal analysis is conducted to define the six modes of resonant frequency for the optimal $z$-stage, as illustrated in Figure 10. The resonant frequency values of six modes from mode 1 to mode 6 were $336.11 \mathrm{~Hz}, 589.53 \mathrm{~Hz}, 594.75 \mathrm{~Hz}$, $596.36 \mathrm{~Hz}, 601.63 \mathrm{~Hz}$, and $605.49 \mathrm{~Hz}$, respectively. In order to avoid the destroy of the optimal $z$-stage, we should evade aforementioned resonant frequency values.
4.5.2. Statistical Analysis. The proficiency of the hybrid approach was assessed with the cuckoo search algorithm [55]. In this article, for a behavior assessment between hybrid approach and CSA, a statistic analysis was exploited by Wilcoxon's rank signed assessment at 5\% substantial grade as well as $95 \%$ guarantee intervals.

Every algorithm was retrieved to achieve optimal results for 35 times with the same constraint conditions. Tables 12 and 13 present the assessment consequences of the Wilcoxon method. It is clear that no significant difference between average worths of the two algorithms is presumed via a null hypothesis. Based on the p-worth consequences (less than 0.05 ), there is a convincing confirmation in opposition to the null hypothesis, as provided in Tables 12 and 13 . Therefore, the behaviors of the proposed algorithm are different to its CSA.

In addition, the Friedman nonparametrics test method was utilized for determining the contrast among the proposed hybrid approach and the CSA at meaningful grade of $\alpha=0.05$. Similarly, every algorithm was retrieved to achieve optimal results for 35 times with the same constraint conditions. The consequences exhibited that the p-worth is smaller than 0.05 . Thus, the null hypothesis would be repudiated. The noteworthy observation was that there is a convincing confirmation in opposition to the null hypothesis, as provided in Tables 14 and 15.

4.5.3. Verification. The optimization values $(M=0.55 \mathrm{~mm}$, $N=0.82377 \mathrm{~mm}, P=0.9 \mathrm{~mm}$, and $K=49 \mathrm{~mm}$ ) would be employed for designing again model of $\mathrm{z}$-stage for simulation confirmation to evaluate the error among the FEA and the combination method. The procedure would be 


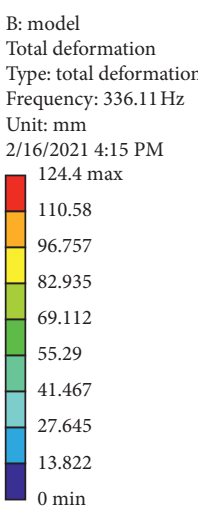

B: model

Total deformation 3

Type: total deformation

Frequency: $594.75 \mathrm{~Hz}$

Unit: $\mathrm{mm}$

2/16/2021 4:16 PM

\begin{tabular}{|l}
$1014.8 \mathrm{~m}$ \\
902.02 \\
789.26 \\
676.51 \\
563.76 \\
451.01 \\
338.26 \\
225.5 \\
112.75 \\
$0 \mathrm{~min}$
\end{tabular}

$0 \mathrm{~min}$

B: model

Total deformation 5

Type: total deformation

Frequency: $601.63 \mathrm{~Hz}$

Unit: $\mathrm{mm}$

2/16/2021 4:17 PM

\begin{tabular}{|}
932.84 \\
829.19 \\
-725.54 \\
621.89 \\
518.24 \\
414.59 \\
310.95 \\
207.3 \\
103.65 \\
$0 \mathrm{~min}$
\end{tabular}

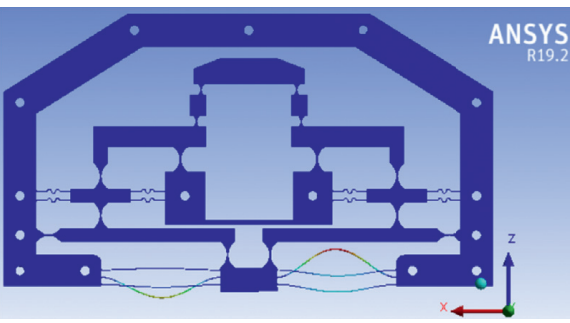

(e)

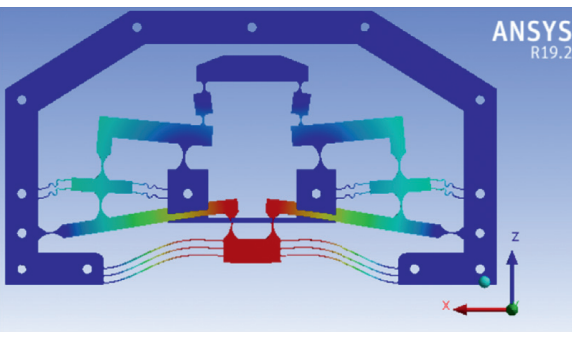

(a)

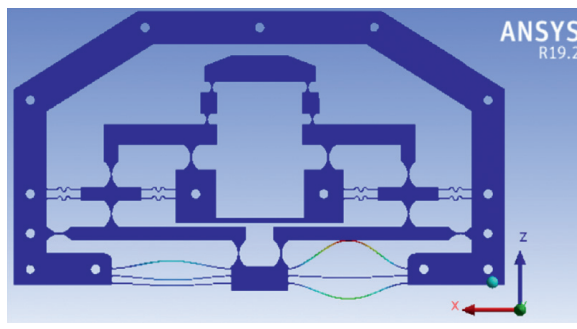

(c)
B: model

Total deformation 2

Type: total deformation

Frequency: $589.53 \mathrm{~Hz}$

Unit: $\mathrm{mm}$

2/16/2021 4:15 PM

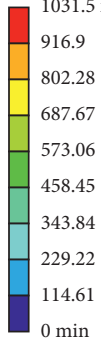

B: model

Total deformation 4

Type: total deformation

Frequency: $596.36 \mathrm{~Hz}$

Unit: $\mathrm{mm}$

2/16/2021 4:16 PM
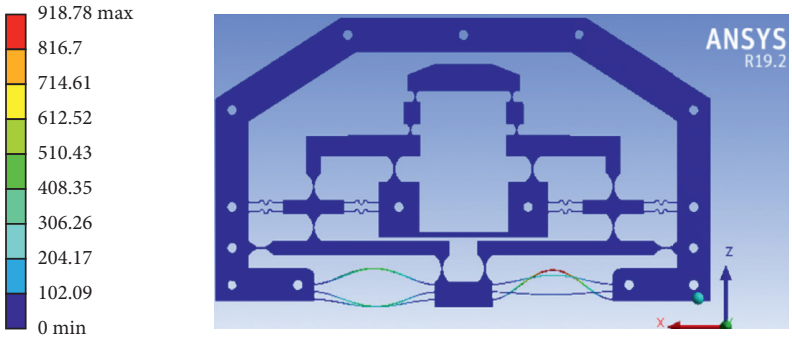

(d)

B: model

Total deformation 6

Type: total deformation

Frequency: $605.49 \mathrm{~Hz}$

Unit: $\mathrm{mm}$

2/16/2021 4:17 PM
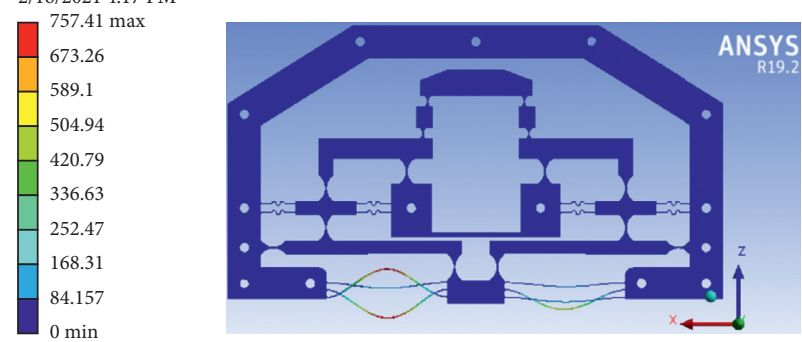

(f)

FIGURE 10: Six modes of resonant natural frequency of the optimal z-stage: (a) Mode 1: resonant frequency of $336.11 \mathrm{~Hz}$; (b) Mode 2: resonant frequency of $589.53 \mathrm{~Hz}$; (c) Mode 3: resonant frequency of $594.75 \mathrm{~Hz}$; (d) Mode 4: resonant frequency of $596.36 \mathrm{~Hz}$; (e) Mode 5 : resonant frequency of $601.63 \mathrm{~Hz}$; (f) Mode 6: resonant frequency of $605.49 \mathrm{~Hz}$.

TABLE 12: Wilcoxon's benchmark of the proposed algorithm vs. CSA for the safety factor.

\begin{tabular}{lccc}
\hline Quantity for assessments & Anticipated medium & P-worth & Wilcoxon statistic \\
\hline 35 & 0.237596 & $\leq 0.001$ & 630 \\
\hline
\end{tabular}

TABLE 13: Wilcoxon's benchmark of the proposed algorithm vs. CSA for the displacement

\begin{tabular}{lccc}
\hline Quantity for assessments & Anticipated medium & P-worth & Wilcoxon statistic \\
\hline 35 & 17.5383 & $\leq 0.001$ & 630 \\
\hline
\end{tabular}


TABLE 14: Friedman tests for the safety factor.

\begin{tabular}{lcc}
\hline Characteristic & Quantity for assessments & Medium \\
\hline$F_{1}$ via proposed algorithm & 35 & 2.17429 \\
$F_{1}$ via CSA & 35 & 2.38246 \\
Total & 70 & 2.27837 \\
DF & Chi-square & P-worth \\
1 & 35 & $\leq 0.001$ \\
Null hypothesis & & $H_{0}:$ all treatment effects are zero \\
Substitute hypothesis & & $H_{1}:$ not all treatment effects are zero \\
\hline
\end{tabular}

TABle 15: Friedman test for the displacement.

\begin{tabular}{lccc}
\hline Characteristic & Quantity for assessments & Medium & Total of ranks \\
\hline$F_{1}$ via proposed algorithm & 35 & 437.583 \\
$F_{1}$ via CSA & 35 & 454.551 \\
Total & 70 & 446.067 \\
DF & Chi-square & P-worth \\
1 & 35 & $\leq 0.001$ \\
Null hypothesis & & $H_{0}:$ all treatment effects are zero \\
Substitute hypothesis & & $H_{1}:$ not all treatment effects are zero \\
\hline
\end{tabular}

TABLE 16: Error between anticipated results and verifications.

\begin{tabular}{lccc}
\hline Characteristics & Anticipation & Confirmation & Error $(\%)$ \\
\hline$F_{1}(\mathrm{~mm})$ & 2.382457 & 2.224 & 7.12 \\
$F_{2}(\mathrm{~mm}$ & 436.04 & 4.25 \\
\hline
\end{tabular}

TABLE 17: Advancement between primary design and optimal design.

\begin{tabular}{lccc}
\hline Characteristics & Primary design & Optimal design & Advancement (\%) \\
\hline$F_{1}(\mathrm{~mm})$ & 2.224 & 2.292 & 3.708 \\
$F_{2}(\mathrm{~mm})$ & 468.076 & 18.498 \\
\hline
\end{tabular}

TABLE 18: Comparison of the proposed z-stage with previous studies.

\begin{tabular}{lcc}
\hline Studies & Dimensions & Output displacement $(\mu \mathrm{m})$ \\
\hline H. Huang et al. [10] & $103 \mathrm{~mm} \times 74 \mathrm{~mm} \times 60 \mathrm{~mm}$ & 11.44 \\
H. Huang et al. [11] & $200 \mathrm{~mm} \times 135 \mathrm{~mm} \times 200 \mathrm{~mm}$ & 40 \\
The proposed z-stage & $301 \mathrm{~mm} \times 162 \mathrm{~mm} \times 16 \mathrm{~mm}$ & 454 \\
\hline
\end{tabular}

accomplished with the same constraints as well as input displacement. Based on the predicted consequences of the hybrid approach, a 3D model was established again for the validation. The output $\mathrm{z}$-displacement was about $436.04 \mu \mathrm{m}$, and the safety factor was around 2.224. It can be clearly seen that that the error among anticipated consequences and verifications for the displacement and safety factor stand at $4.245282 \%$ and $7.124857 \%$, respectively, as depicted in Table 16. Therefore, the anticipated consequences are a trustworthy confirmation with verified consequences.

Additionally, the optimization consequences were better than the results of initial design, as illustrated in Table 17. Moving to a more detailed analysis, an advancement for the safety factor was about $3.708 \%$ as well as its ratio for the displacement was around $18.498 \%$. The consequences demonstrate that the proposed combination approach is a proficient methodology for solving multicriteria optimal problem of the $\mathrm{z}$-stage.
In addition, the proposed $\mathrm{z}$-stage was compared with previous studies, as illustrated in Table 18. Compared with the several commercialized nanoindentation devices, the proposed $\mathrm{z}$-stage has more the output displacement. In addition, this $\mathrm{z}$-stage has small parasitic motion error for ensuring the indenting accuracy.

\section{Conclusions}

This article proposed a novel design of the compliant $\mathrm{z}$-stage integrating the four-lever amplification mechanism, compliant hinge shifted arrangement mechanism, zigzag-based flexure spring guiding mechanism, and symmetric six leaf hinges-based parallel guiding mechanism applied for nano/microindentation testing system and an efficient integration method of the TM, FEM, response surface method, weight factor calculation method based on signal to noise and WOA in order to optimize the key geometrical parameters for improving the essential 
responses of the $\mathrm{Z}$-stage such as the output displacement and the safety factor. For more specifics, the weight factors for both responses were computed via forming the equations chain so as to enhance the calculation precision of optimal results.

First of all, a preliminary design was tested and enhanced by FEA to gain good features according to the merits of a new integrated compliant mechanism of the four-lever amplifier, zigzag guiding mechanism, and parallel guiding mechanism. Then, the numerical experiment data were established by TM. Later on, the output displacement and the safety factor are defined by FEA. Afterward, the regression functions among four design variables and quality responses are formed via exploiting the response surface method. In addition, signal to noise ratios as well as the weight factors for every characteristic are defined. Eventually, according to regression equations, the WOA is executed for determining the optimal parameters.

The results determined that the weight factors are 0.515 for $F_{1}$ and 0.485 for $F_{2}$ where both these values were allocated in the WOA algorithm in order to resolve the multicriteria optimization trouble. The sensitivity analysis and ANOVA were implemented for defining the influences and substantial contributions of input variables on the output characteristics. The consequences revealed that the optimal factors were detected at $M=0.55 \mathrm{~mm}, N=0.82377 \mathrm{~mm}$, $P=0.9 \mathrm{~mm}$, and $K=49 \mathrm{~mm}$. Besides, the results revealed that the optimization safety factor is 2.382456817 as well as the optimization displacement is around $454.5511266 \mathrm{~mm}$.

Based on the results of the Wilcoxon and Friedman nonparametrics testing methods, the proposed algorithm was better than the cuckoo search algorithm. Additionally, the consequences demonstrated that the errors among the optimization consequences and the FEA validations for the safety factor and $z$-axis deformation are $7.12 \%$ and $4.25 \%$, correspondingly. The FEA affirmations were nearby with the anticipated consequences from the integration method. Moreover, the optimization consequences were better than its initial design. For specifics, after optimal process, an advancement for the safety factor and the deformation was around $3.708 \%$ and $18.498 \%$, correspondingly.

In addition, this research meets several challenges such as taking lots of time for creating the great initial design with good performances as well as proposing an effective approach for multiobjective optimization problem. Therefore, topology optimization should be considered to make an initial design of structure, reduce the time consuming, and enhance the characteristics of the design.

In the future study, a prototype will be manufactured and its manners will be checked for assessing with the numerical analysis consequences.

\section{Data Availability}

The data used to support the findings of this study are included within the article.

\section{Conflicts of Interest}

The authors declare that they have no conflicts of interest regarding the publication of this article.

\section{Acknowledgments}

This work belongs to the project grant no. T2020-61TĐ funded by Ho Chi Minh City University of Technology and Education, Vietnam.

\section{References}

[1] J. Nohava, N. X. Randall, and N. Conté, "Novel ultra nanoindentation method with extremely low thermal drift: principle and experimental results," Journal of Materials Research, vol. 24, no. 3, pp. 873-882, 2009.

[2] Z. Hu, K. J. Lynne, S. P. Markondapatnaikuni, and F. Delfanian, "Material elastic-plastic property characterization by nanoindentation testing coupled with computer modeling," Materials Science and Engineering: A, vol. 587, pp. 268-282, 2013.

[3] Q. Xu, "Design and testing of a novel multi-stroke micropositioning system with variable resolutions," Review of Scientific Instruments, vol. 85, no. 2, Article ID 025002, 2014.

[4] K. Lu, J. Zhang, W. Chen, J. Jiang, and W. Chen, "A monolithic microgripper with high efficiency and high accuracy for optical fiber assembly," in Proceedings of the 2014 9th IEEE Conference on Industrial Electronics and Applications, pp. 1942-1947, Hangzhou, China, June 2014.

[5] A. S. Putra, S. Huang, K. K. Tan, S. K. Panda, and T. H. Lee, "Design, modeling, and control of piezoelectric actuators for intracytoplasmic sperm injection," IEEE Transactions on Control Systems Technology, vol. 15, no. 5, pp. 879-890, 2007.

[6] P. Wang and Q. Xu, "Design of a flexure-based constant-force $\mathrm{XY}$ precision positioning stage," Mechanism and Machine Theory, vol. 108, no. 2017, pp. 1-13, 2017.

[7] S. Fan, H. Liu, and D. Fan, "Design and development of a novel monolithic compliant XY stage with centimeter travel range and high payload capacity," Mechanical Sciences, vol. 9, no. 1, pp. 161-176, 2018.

[8] S. P. Wadikhaye, Y. K. Yong, and S. O. R. Moheimani, "Design of a compact serial-kinematic scanner for high-speed atomic force microscopy: an analytical approach," Micro \& Nano Letters, vol. 7, no. 4, pp. 309-313, 2012.

[9] M. Gauthier and E. Piat, "Control of a particular micro-macro positioning system Applied to cell micromanipulation," IEEE Transactions on Automation Science and Engineering, vol. 3, no. 3, pp. 264-271, 2006.

[10] H. Huang, H. Zhao, J. Mi et al., "A novel and compact nanoindentation device for in situ nanoindentation tests inside the scanning electron microscope," AIP Advances, vol. 2, no. 1, Article ID 012104, 2012.

[11] H. Huang, H. Zhao, J. Mi et al., "Experimental research on a modular miniaturization nanoindentation device," Review of Scientific Instruments, vol. 82, no. 9, Article ID 095101, 2011.

[12] W.-L. Zhu, Z. Zhu, P. Guo, and B.-F. Ju, "A novel hybrid actuation mechanism based XY nanopositioning stage with totally decoupled kinematics," Mechanical Systems and Signal Processing, vol. 99, pp. 747-759, 2018.

[13] S. H. Chang and B. C. Du, "A precision piezodriven micropositioner mechanism with large travel range," Review of Scientific Instruments, vol. 69, no. 4, pp. 1785-1791, 1998.

[14] F. Wang, C. Liang, Y. Tian, X. Zhao, and D. Zhang, "Design and control of a compliant microgripper with a large amplification ratio for high-speed micro manipulation," IEEE/ ASME Transactions on Mechatronics, vol. 21, no. 3, pp. 1262-1271, 2016. 
[15] H. Tang, Y. Li, and X. Xiao, "Development and assessment of a novel hydraulic displacement amplifier for piezo-actuated large stroke precision positioning," in Proceedings of the 2013 IEEE International Conference on Robotics and Automation, pp. 1409-1414, Karlsruhe, Germany, May 2013.

[16] Q. Xu, "Structure design of a new compliant gripper based on Scott-Russell mechanism," in Proceedings of the 2013 IEEE International Conference on Robotics and Biomimetics (ROBIO), Shenzhen, China, December 2013.

[17] H.-Y. Kim, D.-H. Ahn, and D.-G. Gweon, "Development of a novel 3-degrees of freedom flexure based positioning system," Review of Scientific Instruments, vol. 83, no. 5, Article ID 055114, 2012.

[18] S. Mirjalili and A. Lewis, "The whale optimization algorithm," Advances in Engineering Software, vol. 95, pp. 51-67, 2016.

[19] J. A. Ghani, I. A. Choudhury, and H. H. Hassan, "Application of Taguchi method in the optimization of end milling parameters," Journal of Materials Processing Technology, vol. 145, no. 1, pp. 84-92, 2004.

[20] C. Kandilli and B. Mertoglu, "Optimisation design and operation parameters of a photovoltaic thermal system integrated with natural zeolite," International Journal of Hydromechatronics, vol. 3, no. 2, pp. 128-139, 2020.

[21] M. Safa, M. Ahmadi, J. Mehrmashadi et al., "Selection of the most influential parameters on vectorial crystal growth of highly oriented vertically aligned carbon nanotubes by adaptive neuro-fuzzy technique selection of the most influential parameters on vectorial crystal growth of highly oriented vertically aligned carbon nanotubes by adaptive neurofuzzy technique," vol. 3, no. 3, pp. 238-251, 2020.

[22] Z. Ali and T. Mahmood, "Complex neutrosophic generalized dice similarity measures and their application to decision making," CAAI Transactions on Intelligence Technology, vol. 5, no. $1,2020$.

[23] B. R. Murlidhar, R. K. Sinha, E. T. Mohamad, R. Sonkar, and M. Khorami, "The effects of particle swarm optimisation and genetic algorithm on ANN results in predicting pile bearing capacity," International Journal of Hydromechatronics, vol. 3, no. 1, pp. 69-87, 2020.

[24] M. Kaur and D. Singh, "Multiobjective evolutionary optimization techniques based hyperchaotic map and their applications in image encryption," Multidimensional Systems and Signal Processing, vol. 32, no. 1, pp. 281-301, 2020.

[25] M. Kaur, D. Singh, and V. Kumar, "Color image encryption using minimax differential evolution-based 7D hyper-chaotic map," Applied Physics B, vol. 126, no. 9, 2020.

[26] H. Golpîra, H. Bevrani, and H. Golpîra, "Application of GA optimization for automatic generation control design in an interconnected power system," Energy Conversion and Management, vol. 52, no. 5, pp. 2247-2255, 2011.

[27] M. Basu and A. Chowdhury, "Cuckoo search algorithm for economic dispatch," Energy, vol. 60, pp. 99-108, 2013.

[28] A. Chander, A. Chatterjee, and P. Siarry, "A new social and momentum component adaptive PSO algorithm for image segmentation," Expert Systems with Applications, vol. 38, no. 5, pp. 4998-5004, 2011.

[29] C. Prakash, S. Singh, M. Singh, K. Verma, B. Chaudhary, and S. Singh, "Multi-objective particle swarm optimization of EDM parameters to deposit HA-coating on biodegradable Mg-alloy," Vacuum, vol. 158, pp. 180-190, 2018.

[30] R. V. Rao and V. Patel, "Multi-objective optimization of heat exchangers using a modified teaching-learning-based optimization algorithm," Applied Mathematical Modelling, vol. 37, no. 3, pp. 1147-1162, 2013.
[31] R. V. Rao, V. J. Savsani, and D. P. Vakharia, "Teachinglearning-based optimization: a novel method for constrained mechanical design optimization problems," Computer-Aided Design, vol. 43, no. 3, pp. 303-315, 2011.

[32] R. V. Rao, V. J. Savsani, and D. P. Vakharia, "Teachinglearning-based optimization: an optimization method for continuous non-linear large scale problems," Information Sciences, vol. 183, no. 1, pp. 1-15, 2012.

[33] N. Le Chau, N. L. Ho, N. T. Tran, and T.-P. Dao, "Analytical model and computing optimization of a compliant gripper for the assembly system of mini direct-current motor," International Journal of Ambient Computing and Intelligence, vol. 12, no. 1, pp. 1-28, 2021.

[34] R. V. Rao and R. B. Pawar, "Constrained design optimization of selected mechanical system components using Rao algorithms," Applied Soft Computing, vol. 89, Article ID 106141, 2020.

[35] T.-H. Hou, C.-H. Su, and W.-L. Liu, "Parameters optimization of a nano-particle wet milling process using the Taguchi method, response surface method and genetic algorithm," Powder Technology, vol. 173, no. 3, pp. 153-162, 2007.

[36] M. P. Dang, H. G. Le, N. Le Chau, and T. Dao, "Optimization for a flexure hinge using an effective hybrid approach of fuzzy logic and moth-flame," Optimization Algorithm, vol. 2021, Article ID 6622655, 2021.

[37] H. Garg, "A hybrid PSO-GA algorithm for constrained optimization problems," Applied Mathematics and Computation, vol. 274, pp. 292-305, 2016.

[38] H. Bhattacharya, S. Chattopadhyay, M. Chattopadhyay, and A. Banerjee, "Storage and bandwidth optimized reliable distributed data allocation algorithm," International Journal of Ambient Computing and Intelligence, vol. 10, no. 1, pp. 78-95, 2019.

[39] N. Le Chau, H. G. Le, T. Dao, M. P. Dang, and V. A. Dang, "Efficient hybrid method of FEA-based RSM and PSO algorithm for multi-objective optimization design for a compliant rotary joint for upper limb assistive device," Mathematical Problems in Engineering, vol. 2019, Article ID 2587373, , 2019.

[40] H. Garg, "A hybrid GSA-GA algorithm for constrained optimization problems," Information Sciences, vol. 478, pp. 499-523, 2019.

[41] H. Garg, "A hybrid GA-GSA algorithm for optimizing the performance of an industrial system by utilizing uncertain data," Handbook of Research on Artificial Intelligence Techniques and Algorithms, pp. 620-654, 2015.

[42] S.-C. Huang and T.-P. Dao, "Multi-objective optimal design of a 2-DOF flexure-based mechanism using hybrid approach of grey-taguchi coupled response surface methodology and entropy measurement," Arabian Journal for Science and Engineering, vol. 41, no. 12, pp. 5215-5231, 2016.

[43] S. Chatterjee, S. Sarkar, S. Hore, N. Dey, A. S. Ashour, and V. E. Balas, "Particle swarm optimization trained neural network for structural failure prediction of multistoried RC buildings," Neural Computing and Applications, vol. 28, no. 8, pp. 2005-2016, 2016.

[44] C. Prakash, H. K. Kansal, B. S. Pabla, and S. Puri, "Multiobjective optimization of powder mixed electric discharge machining parameters for fabrication of biocompatible layer on $\beta$-Ti alloy using NSGA-II coupled with Taguchi based response surface methodology," Journal of Mechanical Science and Technology, vol. 30, no. 9, pp. 4195-4204, 2016.

[45] N. Le Chau, N. T. Tran, and T.-P. Dao, "A multi-response optimal design of bistable compliant mechanism using 
efficient approach of desirability, fuzzy logic, ANFIS and LAPO algorithm," Applied Soft Computing, vol. 94, Article ID 106486, 2020.

[46] T.-P. Dao, "Multiresponse optimization of a compliant guiding mechanism using hybrid taguchi-grey based fuzzy logic approach," Mathematical Problems in Engineering, vol. 2016, Article ID 5386893, 17 pages, 2016.

[47] M. P. Dang, H. G. Le, N. Le Chau, and T. P. Dao, "A multiobjective optimization design for a new linear compliant mechanism," Optimization and Engineering, vol. 21, no. 3, 2020.

[48] D.-N. Nguyen, T.-P. Dao, C. Prakash et al., "Machining parameter optimization in shear thickening polishing of gear surfaces," Journal of Materials Research and Technology, vol. 9, no. 3, pp. 5112-5126, 2020.

[49] H. M. Mohammed, S. U. Umar, and T. A. Rashid, "A systematic and meta-analysis survey of Whale optimization algorithm," Computational Intelligence and Neuroscience, vol. 2019, Article ID 8718571, 2019.

[50] Q. H. Zhai, T. Ye, M. X. Huang, S. L. Feng, and H. Li, "Whale optimization algorithm for multiconstraint second-order stochastic dominance portfolio optimization," Computational Intelligence and Neuroscience, vol. 2020, Article ID 8834162, 2020.

[51] I. Aljarah, H. Faris, and S. Mirjalili, "Optimizing connection weights in neural networks using the whale optimization algorithm," Soft Computing, vol. 22, no. 1, pp. 1-15, 2016.

[52] G. Kaur and S. Arora, "Chaotic whale optimization algorithm," Journal of Computational Design and Engineering, vol. 5, no. 3, pp. 275-284, 2018.

[53] T.-P. Dao, S.-C. Huang, and N. Le Chau, "Robust parameter design for a compliant microgripper based on hybrid Taguchidifferential evolution algorithm," Microsystem Technologies, vol. 24, no. 3, pp. 1461-1477, 2017.

[54] A. C. Analysis, "Statistical study of machine learning algorithms using parametric and non-parametric tests," International Journal of Ambient Computing and Intelligence, vol. 11, no. 3, pp. 80-105, 2020.

[55] A. H. Gandomi, X.-S. Yang, and A. H. Alavi, "Cuckoo search algorithm: a metaheuristic approach to solve structural optimization problems," Engineering with Computers, vol. 29, no. 1, pp. 17-35, 2013. 